\title{
INTERAÇÕES MEDICAMENTOSAS POTENCIAIS EM PACIENTES HOSPITALIZADOS
}

\author{
POTENTIAL DRUG INTERACTIONS IN HOSPITALIZED PATIENTS
}

Aniele Aparecida Petri ${ }^{a^{*}}$

Orcid: https://orcid.org/0000-0002-1045-647X

Aline Schneider ${ }^{b^{*}}$

Orcid: https://orcid.org/0000-0003-0438-6599

Karine Raquel Uhdich Kleibert ${ }^{*}$

Orcid: https://orcid.org/0000-0001-7511-1977
Vivian Lemes Lobo Bittencourt ${ }^{\mathrm{d}^{* *}}$
Orcid: https://orcid.org/0000-0003-1488-0611

Eliane Roseli Winkelmann ${ }^{\mathrm{e}^{*}}$

Orcid: https://orcid.org/0000-0002-4922-6516

Christiane de Fátima Colet ${ }^{\mathrm{f}^{*}}$

Orcid: https://orcid.org/0000-0003-2023-5088

anielepetri@gmail.com ${ }^{\mathrm{a}}$, aline_schneider90@hotmail.com ${ }^{\mathrm{b}}$, karine.u.k@hotmail.com , vivillobo@hotmail.com ${ }^{\mathrm{d}}$,elianew@unijui.edu.br christiane.colet@unijui.edu.br ${ }^{\mathrm{f}}$

Universidade Regional do Noroeste do Estado do Rio Grande do Sul - UNIJUÍ*, Universidade Regional Integrada do Alto Uruguai e das Missões -URI, Santo Ângelo**

Data de Submissão: 23/08/2019

Data de Aceite: 18/12/2019

\section{RESUMO}

Introdução: No ambiente hospitalar é comum para a prática clínica o uso de muitos medicamentos, o que aumenta o risco da ocorrência de potenciais interações medicamentosas. Objetivo: Descrever as interações medicamentosas potenciais nas prescrições de unidades de internação hospitalar. Métodos: Trata-se de um estudo quantitativo, transversal, descritivo realizado em unidades de internação clínica e cirúrgica de um hospital privado. Os dados dos medicamentos foram coletados a partir do prontuário e classificados pela Anatomical Therapeutic Chemical. As interações medicamentosas potenciais foram checadas no software Micromedex. Resultados: A amostra foi constituída por 612 indivíduos, 324 pacientes cirúrgicos e 288 clínicos. Houve predomínio do sexo feminino, idade média de 55 anos com variação de 18 a 100 anos, $29,1 \%$ possuíam ensino fundamental incompleto, $96,2 \%$ da raça branca e $62,4 \%$ casados. O medicamento mais prescrito foi a morfina, seguido do enalapril. As interações medicamentosas classificadas como graves totalizaram 54,8\%. Os fatores associados com apresentar interações medicamentosas foram: apresentar hipertensão, uso de anti-hipertensivos, em especial as classes farmacológicas beta-bloqueadores e inibidores da ECA; uso de psicoanalépticos, psicoléptico e corticoides sistêmicos. Conclusões: No presente estudo, a potencial interação medicamentosa mais frequente foi entre a morfina e o tramadol, a média de interações medicamentosas foi de 3,6 $\pm 4,9 /$ paciente. Destacou-se a necessidade da avaliação do uso de medicamentos durante a internação hospitalar e suas potenciais interações medicamentosas, visando minimizar os riscos relacionados ao uso de medicamentos e aumentando a segurança do paciente internado.

Palavras-chave: Interações medicamentosas; medicamentos; hospital; sistema nervoso; anti-hipertensivos.

\section{ABSTRACT}

Introduction: No hospital environment is common for clinical practice or use of many medications, which leads to the risk of drug interactions occurring. Objective: To describe how drug interactions are used in prescriptions of inpatient units. Methods: This is a quantitative, cross-sectional, descriptive study conducted in clinical and surgical inpatient units of a private hospital. Drug data were collected from medical records and classified by Anatomical Therapeutic Chemical. The drug interactions used were not checked in the Micromedex software. Results: The sample was used by 612 individuals, 324 surgical patients and 288 clinicians. There was a predominance of females, mean age 55 years ranging from 18 to 100 years, $29.1 \%$ had incomplete elementary school, $96.2 \%$ white and $62.4 \%$ married. The most commonly prescribed drug was morphine, followed by enalapril. Drug interactions classified as graves total $54.8 \%$. Factors associated with drug presentations were: hypertension presentation, use of antihypertensive drugs, especially as beta-blocker and ACE inhibitor drug classes; use of psychoanalytical, psychological and systemic corticosteroids. Conclusions: In the present study, the most frequent drug interaction potential was between morphine and tramadol, the average drug interaction was $3.6 \pm 4.9$ / patient. Avoid the need to evaluate medication use during a hospital stay and its drug interactions, minimize the risks related to medication use, and use inpatient safety.

Keywords: Drug interactions; drugs; hospitals; nervous system; antihypertensive agents. 


\section{Introdução}

A associação de medicamentos é comum na prática clínica e se justifica pelo efeito terapêutico sinérgico, aumento da eficácia do tratamento e é necessária especialmente na terapia de múltiplas doenças coexistentes ${ }^{(1)}$. A polifarmácia está associada com a baixa adesão ao tratamento, maior risco de erros de medicação, aumento da taxa de hospitalização, problemas relacionados a medicamentos, entre esses as interações medicamentosas $(\mathrm{IM})^{(2,3)}$, aumento de eventos adversos e mortalidade ${ }^{(4)}$.

As estimativas de ocorrência de IM estão entre 3 a 5\% em pacientes que fazem uso de menos de cinco fármacos e $20 \%$ entre aqueles que fazem uso de 10 a 20 fármacos simultaneamente ${ }^{(5)}$. O estudo sobre as IM e sua relação direta com a utilização de polifarmácia ganha maior importância na área médica, em especial no âmbito hospitalar ${ }^{(6)}$.

Em estudo realizado por Santos (2017), em um hospital universitário no Rio de Janeiro, com pacientes internados em uma unidade de terapia intensiva, $74 \%$ dos pacientes estiveram expostos a interações medicamentosas potenciais durante o tempo de permanência na UTI. Foram identificados IM em $72,7 \%$ das prescrições, o que correspondeu a uma média de 2,96 interações por prescrição, variando de 1 a 13 interações potenciais. Dos pacientes, $6,8 \%$ apresentaram evento adverso decorrente de IM, entre esses efeitos estavam: hipotensão, bradicardia, hemorragia, hepatotoxidade, rash cutâneo, náusea e convulsão. Os pacientes mais expostos as IM eram os que tinham tempo de internação na UTI significativamente mais elevado e os com mediana de medicamentos diferentes administrados na internação significativamente superior ${ }^{(7)}$

Em um estudo transversal que avaliou as prescrições de pacientes da oncologia pediátrica de um hospital, em $87,5 \%$ das prescrições havia a possibilidade de ocorrência de alguma incompatibilidade e foram encontradas 2108 incompatibilidades medicamentosas ao total, sendo 300 diferentes combinações entre dois medicamentos ${ }^{(8)}$. Entretanto, no Brasil, ainda são poucos os estudos que têm demonstrado a frequência das IM em população hospitalizada.

Muitas dessas interações apresentam manifestações clínicas de início lento e podem ser erroneamente interpretadas como novas doenças, o que dificulta o manejo adequado ${ }^{(9)}$.

Neste contexto, ao considerar que os medicamentos são parte essencial na terapêutica do paciente no ambiente hospitalar, o objetivo deste estudo é descrever as IM potenciais nas prescrições de unidades de internação hospitalar.

\section{Metodologia}

Trata-se de um estudo quantitativo, transversal, descritivo, realizado em unidades de internação clínica e cirúrgica de um hospital privado, de médio porte, na região noroeste do Estado do Rio Grande do Sul. A instituição possui 115 leitos, destes 79 compuseram o cenário do estudo. Os participantes foram internados nessas unidades no período de 1 de junho a 17 de agosto de 2015 . Um total de 627 pacientes foram convidados, oito recusaram, seis foram hospitalizados mais de uma vez durante o período do estudo e um foi excluído por dados incompletos, correspondentes a 612 pacientes. Os critérios de inclusão foram: pacientes com idade superior a 18 anos, internados em unidades clínicas ou cirúrgicas dentro de um período de até 24 horas. Os critérios de exclusão foram: pacientes hospitalizados mais de uma vez no período do estudo. Demais dados sobre a seleção dos participantes está descrito em Bittencourt et al (2017) ${ }^{(10)}$.

Os dados dos medicamentos foram coletados a partir do prontuário para posterior análise. Os medicamentos foram classificados pelo primeiro, segundo e quinto níveis da classificação Anatomical Therapeutic Chemical ${ }^{(11)}$.

Para a verificação das IM potenciais utilizou-se o software Micromedex ${ }^{\oplus(12)}$ e estas foram classificadas quanto a gravidade em: contraindicada, grave, moderada e menor. Os medicamentos não encontrados nessa base de dados foram pesquisados em Porto, Jacomini e Silva $(2010)^{(13)}$. As interações foram classificadas também quanto ao sistema fisiológico afetado pela IM e os efeitos gerados em decorrência.

Interação contraindicada é aquela em que o uso concomitante das medicações não é aconselhado. Interação grave pode representar perigo à vida 
e requer intervenção médica para diminuir ou evitar efeitos adversos graves. Interação moderada pode resultar em exacerbação do problema de saúde do paciente e requer alteração no tratamento. Interação menor resultará em efeitos clínicos limitados, as manifestações podem incluir aumento na frequência ou gravidade dos efeitos colaterais, porém geralmente não requerem uma alteração importante no tratamento ${ }^{(6)}$. Para fins de análise as interações foram classificadas quanto ao sistema que ela afeta.

Os dados obtidos foram compilados em tabelas por meio do software StatisticalPackage for the Social Sciences (versão 18.0), sendo realizada análise descritiva simples com média frequência, desvio padrão e estatística analítica.

Este estudo foi aprovado pelo Comitê de Ética em Pesquisa da Universidade Regional do Noroeste do Estado do Rio Grande do Sul UNIJUÍ, com parecer consubstanciado número 1.078.277/2015 e CAAE 43893615.6.0000.5350.

\section{Resultados}

A amostra foi constituída por 612 indivíduos, 324 pacientes cirúrgicos $(52,9 \%)$ e 288 clínicos $(47,1 \%)$. Houve predomínio do sexo feminino $(62,3 \%)$, idade média de 55 anos com variação de 18 a 100 anos, 29,1\% possuíam Ensino Fundamental Incompleto, 96,2\% da raça branca e $62,4 \%$ casados.

A Tabela 1 apresenta os dados dos medicamentos, segundo a classificação ATC, nível 1, tendo como classe de medicamentos mais citada o Sistema Cardiovascular, e o principal medicamento o Enalapril, seguido pelo Sistema Nervoso, com maior frequência da Morfina, e em terceiro o Sistema Digestório e Metabólito, sendo o medicamento mais frequente a Metoclopramida.

Tabela 1 - Classificação dos medicamentos segundo ATC, primeiro e quinto nível - de pacientes hospitalizados. Ijuí, RS, Brasil, 2015

\begin{tabular}{c|c|c}
\hline $\begin{array}{c}\text { Classe farmacêutica - } \\
\text { ATC 1 }\end{array}$ & nedicamento - ATC $\mathbf{~}$ & $\mathbf{1}$ \\
\hline & Enalapril & $126(16,1 \%)$ \\
Sistema Cardiovascular & Captopril & $104(13,2 \%)$ \\
& Hidroclorotiazida & $77(9,8 \%)$ \\
& Furosemida & $37(4,7 \%)$ \\
& Losartana & $30(3,8 \%)$ \\
& Outros & $110(14,1 \%)$ \\
\hline \multirow{2}{*}{ Sistema Nervoso } & Morfina & $202(25,8 \%)$ \\
& Diazepam & $68(8,7 \%)$ \\
& Tramadol & $59(7,5 \%)$ \\
& Clonazepam & $41(5,24 \%)$ \\
& Alprazolam & $34(4,3 \%)$ \\
& Outros & $277(35,4 \%)$ \\
\hline \multirow{2}{*}{ Sistema Digestório e Metabólito } & Metoclopramida & $91(11,6 \%)$ \\
& Omeprazol & $82(10,5 \%)$ \\
& Metformina & $45(5,75 \%)$ \\
& Glibenclamida & $16(2,0 \%)$ \\
& Glimepirida & $7(0,9 \%)$ \\
& Outros & $7(0,9 \%)$ \\
\hline \multirow{2}{*}{ Sistema Musculoesquelético } & Ciclobenzaprina & $18(2,3 \%)$ \\
& Tizanidina & $1(0,13 \%)$ \\
& Ibuprofeno & $1(0,13 \%)$ \\
\hline
\end{tabular}




\begin{tabular}{|c|c|c|}
\hline Anti-infeccioso para uso Sistêmico & $\begin{array}{l}\text { Levofloxacino } \\
\text { Fluconazol } \\
\text { Ciprofloxacino } \\
\text { Azitromicina } \\
\text { Eritromicina }\end{array}$ & $\begin{array}{c}14(0,18 \%) \\
6(0,77 \%) \\
3(0,4 \%) \\
2(0,25 \%) \\
2(0,25 \%)\end{array}$ \\
\hline Sistema Respiratório & Fluticasona & $1(0,13 \%)$ \\
\hline $\begin{array}{l}\text { Preparações Hormônicas Sistemas, } \\
\text { excl. Hormonas sexuais e insulinas }\end{array}$ & $\begin{array}{l}\text { Insulina Regular } \\
\text { Insulina Lispro } \\
\text { Insulina } \\
\text { Insulina Glargina } \\
\text { Insulina NPH } \\
\text { Outros }\end{array}$ & $\begin{array}{c}31(3,96 \%) \\
11(1,40 \%) \\
8(1,0 \%) \\
7(0,9 \%) \\
4(0,5 \%) \\
7(0,9 \%)\end{array}$ \\
\hline $\begin{array}{l}\text { Sistema Cardiovascular Agentes de } \\
\text { Modificação de Lipídeos }\end{array}$ & $\begin{array}{c}\text { Sinvastatina } \\
\text { Rosuvastatina }\end{array}$ & $\begin{array}{c}11(1,40 \%) \\
1(0,13 \%)\end{array}$ \\
\hline $\begin{array}{c}\text { Órgãos de Fornecimento de Sangue e } \\
\text { sangue }\end{array}$ & Varfarina & $2(0,25 \%)$ \\
\hline Dermatológicos & $\begin{array}{c}\text { Prometazina } \\
\text { Cetoconazol } \\
\text { Difenidramina }\end{array}$ & $\begin{array}{l}6(0,77 \%) \\
2(0,25 \%) \\
2(0,25 \%)\end{array}$ \\
\hline Outros & $\begin{array}{c}\text { Ginko } \\
\text { Alantoína } \\
\text { Meclizina } \\
\text { Dimenidrato } \\
\text { Alantoina + Aminacride }+ \\
\text { Sulfanilamida (cloridrato) }\end{array}$ & $\begin{array}{l}4(0,5 \%) \\
4(0,5 \%) \\
1(0,13 \%) \\
1(0,13 \%) \\
1(0,13 \%)\end{array}$ \\
\hline
\end{tabular}

${ }^{1}$ Percentual relacionado ao número de ocorrências de interações com o medicamento relacionado ao número total de interações (782).

Fonte: Dados da pesquisa

A Tabela 2 apresenta as IM classificadas de acordo com a gravidade, sendo que a mais frequente $(7,7 \%)$ entre a Morfina e o Tramadol.
A média de interações medicamentosas foi de $3,6 \pm 4,9 /$ paciente, e a média de medicamentos prescritos foi $3,1 \pm 1,8 /$ paciente.

Tabela 2 - Interações medicamentosas classificadas de acordo com a gravidade e ocorrência em de pacientes hospitalizados. Ijuí, RS, Brasil, 2015.

\begin{tabular}{c|c|c|c}
\hline Medicamento 1 & Medicamento 2 & $\mathbf{N}^{\mathbf{0}}$ de ocorrências $\left.\mathbf{( \% \mathbf { 1 }}\right)$ & Gravidade da interação \\
\hline \multirow{6}{*}{ Morfina } & Tramadol & $47(7,7)$ & Maior \\
& Captopril & $35(5,7)$ & Maior \\
& Clonazepam & $33(5,4)$ & Maior \\
& Diazepam & $19(3,1)$ & Maior \\
& Ciclobenzaprina & $18(2,9)$ & Maior \\
& Lorazepam & $9(1,5)$ & Maior \\
& Codeina & $7(1,1)$ & Maior \\
& Clorpromazina & $6(1,0)$ & Maior \\
& Alprazolam & $4(0,7)$ & Maior \\
\hline
\end{tabular}




\begin{tabular}{c|c|c|c}
\hline & Citalopram & $18(2,9)$ & Contraindicada \\
& Amitriptilina & $9(1,5)$ & Contraindicada \\
& Clorpromazina & $9(1,5)$ & Contraindicada \\
& Paroxetina & $9(1,5)$ & Contraindicada \\
& Haloperidol & $8(1,3)$ & Contraindicada \\
& Duloxetina & $6(1,0)$ & Contraindicada \\
& Quetiapina & $6(1,0)$ & Maior \\
& Fluoxetina & $4(0,7)$ & Maior \\
& Losartana & $12(2,0)$ & Maior \\
\cline { 1 - 3 } & Espironolactona & $4(0,7)$ & Maior \\
\cline { 1 - 3 } & Espironolactona & $9(1,5)$ & Maior \\
\hline Enalapril & Paroxetina & $5(0,8)$ & Maior \\
\hline Tramadol & Duloxetina & $4(0,7)$ & Maior \\
\hline Citalopram & Codeina & $4(0,7)$ & Menor, Moderada, Maior, \\
\hline Clonazepam & Outras & $500(81,7)$ & ser avaliado \\
\hline \multirow{2}{*}{ Outras* } & & & \\
\hline
\end{tabular}

1 Percentual relacionado ao número de pacientes do estudo $(\mathrm{n}=612)$

* Frequência $<0,7 \%$ foram incluídas na categoria outras Fonte: Dados da pesquisa.

Considerando o perfil de gravidade das 1.672 IM observadas, verificou-se que, $54,8 \%$ das IM foram graves, $39,5 \%$ moderadas e $5,7 \%$ IM menores. Em relação às IM associadas a efeitos depressores do sistema nervoso central, $17(2,5 \%)$ estavam relacionadas à morfina, e $8(1,3 \%)$ ao alprazolam, conforme descrito na Tabela 3 . Quanto às IM com risco de efeitos extrapiramidais, $18(2,6 \%)$ estavam associadas à metoclopramida. Em relação ao risco de rabdomiólise, o maior número de IM estava associado ao medicamento sinvastatina (1\%).

Tabela 3 - Descrição qualitativa das Interações medicamentosas e efeito associado ao Sistema fisiológico em pacientes hospitalizados. Ijuí, RS, Brasil, 2015.

\begin{tabular}{c|c|c|c}
\hline $\begin{array}{c}\text { SISTEMA AFETADO } \\
\text { PELA IM }\end{array}$ & $\begin{array}{c}\text { EFEITO } \\
\text { DESENVOLVIDO } \\
\text { PELA IM }\end{array}$ & MEDICAMENTO 1 & $\begin{array}{c}\text { MEDICAMENTO 2 } \\
\text { SNC }\end{array}$ \\
\hline DEPRESSOR \\
DO SNC
\end{tabular}




\begin{tabular}{|c|c|c|c|}
\hline $\begin{array}{l}\text { SISTEMA AFETADO } \\
\text { PELA IM }\end{array}$ & $\begin{array}{l}\text { EFEITO } \\
\text { DESENVOLVIDO } \\
\text { PELA IM }\end{array}$ & MEDICAMENTO 1 & MEDICAMENTO 2 \\
\hline \multirow{19}{*}{$\mathrm{SNC}$} & \multirow{14}{*}{$\begin{array}{l}\text { DEPRESSOR } \\
\text { DO SNC }\end{array}$} & Amitriptilina & Levotiroxina \\
\hline & & Tramadol & $\begin{array}{c}\text { Sertralina, fluconazol, fluoxetina, } \\
\text { eritromicina }\end{array}$ \\
\hline & & Alprazolam & $\begin{array}{l}\text { Zolpidem, fluoxetina, sertralina, } \\
\text { fluconazol, mirtazapina, carbamazepina, } \\
\text { haloperidol, diltiazem }\end{array}$ \\
\hline & & Clonazepam & Zolpidem, fluconazol, cetoconazol \\
\hline & & Carbonato de lítio & Losartana, hidroclortiazida \\
\hline & & Carbamazepina & $\begin{array}{l}\text { Risperidona, quetiapina, fluconazol, } \\
\text { azitromicina, ibuprofeno }\end{array}$ \\
\hline & & Clorpromazina & Zolpidem, atenolol, fluoxetina \\
\hline & & Lorazepam & Olanzapina, zolpidem \\
\hline & & Zolpidem & Sertralina, venlafaxina \\
\hline & & Fenitoina & Risperidona \\
\hline & & Mirtazapina & Midazolam, duloxetina \\
\hline & & Imipramina & Venlafaxina \\
\hline & & Divalproato de Sódio & Lamotrigina \\
\hline & & Sertralina & Haloperidol \\
\hline & $\begin{array}{c}\text { EFEITOS } \\
\text { EXTRAPIRAMIDAIS }\end{array}$ & Metoclopramida & $\begin{array}{l}\text { Citalopram, amitriptilina, paroxetina, } \\
\text { clorpromazina, haloperidol, duloxetina, } \\
\text { quetiapina, fluoxetina, prometazina, } \\
\text { risperidona, venlafaxina, sertralina, } \\
\text { tioridazina, bupropiona, escitalopram, } \\
\text { carbidopalevodopa, trazodona, } \\
\text { imipramina, }\end{array}$ \\
\hline & & Ciclobenzaprina & Morfina \\
\hline & AUMENTO RISCO & Dipirona & Levofloxacino \\
\hline & DE CONVULSÃO & Tramadol & Paroxetina, risperidona, tioridazida \\
\hline & & Bupropiona & Dimenidrato \\
\hline \multirow{8}{*}{ CARDIOVASCULAR } & \multirow{8}{*}{ HIPOTENSÃO } & Enalapril & $\begin{array}{l}\text { Hidroclorotiazida, Furosemida, } \\
\text { espironolactona, losartana, clortalidona, } \\
\text { Amilorida + Hidroclorotiazida }\end{array}$ \\
\hline & & Captopril & $\begin{array}{l}\text { Losartana, furosemida, hidroclortiazida, } \\
\text { clorpromazina, espironolactona, } \\
\text { clortalidona, valsartana }\end{array}$ \\
\hline & & Hidralazina & Furosemida, tartarato de metoprolol \\
\hline & & Amiodarona & $\begin{array}{l}\text { Metoprolol, ginko, atenolol, anlodipino, } \\
\text { carvedilol }\end{array}$ \\
\hline & & Metoprolol & Paroxetina, clorpromazina, duloxetina, \\
\hline & & Losartana & Fluconazol, espironolactona, amiodarona \\
\hline & & Furosemida & Fluticasona, hidrocortisona, ramipril \\
\hline & & $\begin{array}{l}\text { Atenolol }+ \\
\text { Clortalidona }\end{array}$ & Cálcio + Colecalciferol \\
\hline
\end{tabular}




\begin{tabular}{|c|c|c|c|}
\hline $\begin{array}{c}\text { SISTEMA AFETADO } \\
\text { PELA IM }\end{array}$ & $\begin{array}{c}\text { EFEITO } \\
\text { DESENVOLVIDO } \\
\text { PELA IM } \\
\end{array}$ & MEDICAMENTO 1 & MEDICAMENTO 2 \\
\hline CARDIOVASCULAR & HIPOTENSÃO & $\begin{array}{l}\text { Hidroclortiazida } \\
\text { Anlodipino } \\
\text { Propanolol } \\
\text { Ramipril } \\
\text { Clonidina }\end{array}$ & $\begin{array}{c}\text { Gluconato de cálcio, Ramipril e Amilorida } \\
\text { (cloridrato), Captopril e Amilorida } \\
\text { (cloridrato), predinisolona } \\
\text { Carvedilol, atenolol, } \\
\text { Amitriptilina, omeprazol } \\
\text { Espironolactona } \\
\text { Quetiapina }\end{array}$ \\
\hline MUSCULAR & RABDOMIOLISE & $\begin{array}{l}\text { Sinvastatina } \\
\text { Rosuvastatina }\end{array}$ & $\begin{array}{c}\text { Varfarina, risperidona, Pantotenato de } \\
\text { sódio + Cyanocobalamin + Inositol+ } \\
\text { Niacina + Piridoxina + Riboflavina }+ \\
\text { Tiamina, cetoconazol, azitromicina, } \\
\text { amiodarona } \\
\text { Fluconazol }\end{array}$ \\
\hline ENDÓCRINO & HIPOGLICEMIA & $\begin{array}{l}\text { Metformina } \\
\text { Gliblenclamida } \\
\text { Glimepirida } \\
\text { Sitagliptina }\end{array}$ & $\begin{array}{c}\text { Metformina, enalapril, losartana, captopril, } \\
\text { levofloxacino, glimepirida, ginko, } \\
\text { metoprolol, alantoina, ciprofloxacino, } \\
\text { clonidina, Alantoina + Aminacride + } \\
\text { Sulfanilamida (cloridrato), } \\
\text { Enalapril, captopril, metoprolol, } \\
\text { levofloxacino, ciprofloxacino, Dipirona + } \\
\text { Escopolamina, } \\
\text { Omeprazol, enalapril, dipirona, } \\
\text { sinvastatina, atenolol, aas, metoprolol, } \\
\text { prednisona, } \\
\text { Captopril, metoprolol, carvedilol, enalapril } \\
\text { Ciprofloxacino }\end{array}$ \\
\hline
\end{tabular}

Fonte: Dados da pesquisa.

A Tabela 4 apresenta a análise dos fatores associados às IM. Observa-se que os pacientes hipertensos estavam mais suscetíveis à IM $(\mathrm{p}=0,006)$. Além de hipertensão, houve associação com uso de anti-hipertensivos ( $\mathrm{p}=0,044)$, em especial as classes farmacológicas beta-bloqueadores $(p=0,04)$ e inibidores da ECA $(p=0,022)$. Outras classes farmacológicas que apresentaram associação com IM foram: psicoanalépticos $\quad(\mathrm{p}=0,003), \quad$ psicoléptico $(\mathrm{p}=0,000)$ e corticoides sistêmicos $(\mathrm{p}=0,049)$. As demais variáveis apresentadas na tabela não apresentaram significância estatística.
Tabela 4 - Relação entre variáveis sociodemográficas, clínicas e medicamentosas e a presença de interações medicamentosas. Ijuí, RS, Brasil, 2015.

\begin{tabular}{|c|c|c|c|c|}
\hline \multicolumn{5}{|c|}{ Interação medicamentosa } \\
\hline & $\begin{array}{c}\text { Sim } \\
n(\%)\end{array}$ & $\begin{array}{l}\text { Não } \\
n(\%)\end{array}$ & $P$ & $\begin{array}{c}\text { Razão } \\
\text { de risco }\end{array}$ \\
\hline \multicolumn{5}{|c|}{ Idade } \\
\hline $\begin{array}{l}\text { Menos de } \\
60 \text { anos }\end{array}$ & $213(54,6)$ & $129(58,1)$ & \multirow{2}{*}{0,403} & \multirow{2}{*}{0,868} \\
\hline $\begin{array}{c}\text { Mais de } 60 \\
\text { anos }\end{array}$ & $177(45,4)$ & $93(41,9)$ & & \\
\hline \multicolumn{5}{|c|}{ Sexo } \\
\hline Masculino & $142(36,4)$ & $89(40,1)$ & \multirow{2}{*}{0,367} & \multirow{2}{*}{0,856} \\
\hline Feminino & $248(63,6)$ & $133(59,9)$ & & \\
\hline \multicolumn{5}{|c|}{ Tipo de Unidade de Internação } \\
\hline Clínica & $194(49,7)$ & $94(42,3)$ & \multirow{2}{*}{0,078} & \multirow{2}{*}{1,348} \\
\hline Cirúrgica & $196(50,3)$ & $128(57,7)$ & & \\
\hline
\end{tabular}




\begin{tabular}{|c|c|c|c|c|}
\hline \multicolumn{5}{|c|}{ Interação medicamentosa } \\
\hline \multicolumn{5}{|c|}{ Comorbidades } \\
\hline \multicolumn{5}{|c|}{$H A S$} \\
\hline Sim & $165(42,3)$ & $69(31,1)$ & \multirow{2}{*}{$0,006^{*}$} & \multirow{2}{*}{1,626} \\
\hline Não & $225(57,7)$ & $153(68,9)$ & & \\
\hline \multicolumn{5}{|c|}{ Diabetes } \\
\hline Sim & $42(10,8)$ & $22(9,9)$ & \multirow{2}{*}{0,738} & \multirow{2}{*}{1,097} \\
\hline Não & $348(89,2)$ & $200(90,1)$ & & \\
\hline \multicolumn{5}{|c|}{ Tratamento } \\
\hline \multicolumn{5}{|c|}{ Betabloqueadores } \\
\hline Sim & $58(14,9)$ & $22(9,9)$ & \multirow{2}{*}{$0,040 *$} & \multirow{2}{*}{1,588} \\
\hline Não & $332(85,1)$ & $200(90,1)$ & & \\
\hline \multicolumn{5}{|c|}{ Anti-hipertensivos } \\
\hline Sim & $17(4,4)$ & $3(1,4)$ & \multirow{2}{*}{$0,044^{*}$} & \multirow{2}{*}{3,327} \\
\hline Não & $373(95,6)$ & $219(98,6)$ & & \\
\hline \multicolumn{5}{|c|}{ Bloqueadores de canais de cálcio } \\
\hline Sim & $19(4,9)$ & $9(4,1)$ & \multirow{2}{*}{0,642} & \multirow{2}{*}{1,212} \\
\hline Não & $371(95,1)$ & $213(95,9)$ & & \\
\hline \multicolumn{5}{|c|}{ Renina angiotensina } \\
\hline Sim & $135(34,6)$ & $57(25,7)$ & \multirow{2}{*}{$0,022 *$} & \multirow{2}{*}{1,533} \\
\hline Não & $255(65,4)$ & $165(74,3)$ & & \\
\hline \multicolumn{5}{|c|}{ Diurético } \\
\hline Sim & $79(20,3)$ & $33(14,9)$ & \multirow{2}{*}{0,097} & \multirow{2}{*}{1,455} \\
\hline Não & $311(79,7)$ & $189(85,1)$ & & \\
\hline \multicolumn{5}{|c|}{ Terapia cardíaca } \\
\hline Sim & $9(2,3)$ & $1(0,5)$ & \multirow{2}{*}{0,081} & \multirow{2}{*}{5,220} \\
\hline Não & $381(97,7)$ & $222(99,5)$ & & \\
\hline & $P S$ & coanaléptic & & \\
\hline Sim & $90(23,1)$ & $29(13,1)$ & & 1997 \\
\hline Não & $300(76,9)$ & $193(86,9)$ & 0,003 & 1,991 \\
\hline & & sicolépticos & & \\
\hline Sim & $147(37,7)$ & $53(23,9)$ & $0000 *$ & 1929 \\
\hline Não & $243(62,3)$ & $169(76,1)$ & 0,000 & 1,929 \\
\hline & & ntiepiléticos & & \\
\hline Sim & $67(17,2)$ & $34(15,3)$ & 0550 & 11147 \\
\hline Não & $323(82,8)$ & $188(84,7)$ & U, & 1,1141 \\
\hline & & Opióides & & \\
\hline Sim & $\begin{array}{c}210 \\
(53,8 \%)\end{array}$ & $\begin{array}{c}112 \\
(50,5 \%)\end{array}$ & 0410 & 1146 \\
\hline Não & $\begin{array}{c}180 \\
(46,2 \%)\end{array}$ & $\begin{array}{c}110 \\
(49,5 \%)\end{array}$ & 0,419 & 1,140 \\
\hline
\end{tabular}

\begin{tabular}{|c|c|c|c|c|}
\hline \multicolumn{5}{|c|}{ Interação medicamentosa } \\
\hline \multicolumn{5}{|c|}{ Outros analgésicos e antipiréticos } \\
\hline Sim & $333(85,4)$ & $181(81,5)$ & \multirow{2}{*}{0,211} & \multirow{2}{*}{1,323} \\
\hline Não & $57(14,6)$ & $41(18,5)$ & & \\
\hline \multicolumn{5}{|c|}{ Corticoesteroides sistêmicos } \\
\hline Sim & $50(12,8)$ & $17(7,7)$ & \multirow{2}{*}{$0,049^{*}$} & \multirow{2}{*}{1,773} \\
\hline Não & $340(87,2)$ & $205(92,3)$ & & \\
\hline \multicolumn{5}{|c|}{ Hipoglicemiantes } \\
\hline Sim & $39(10)$ & $18(8,1)$ & \multirow{2}{*}{0,439} & \multirow{2}{*}{1,259} \\
\hline Não & $351(90)$ & $204(91,9)$ & & \\
\hline
\end{tabular}

a. 0 células $(0,0 \%)$ esperavam uma contagem menor que 5 . A contagem mínima esperada é 97,94 .

b. Computado apenas para uma tabela $2 \times 2$

DP: desvio padrão; n: número; \%: percentual; valor de $\mathrm{p}$ para o teste de qui-quadrado de Pearson; ${ }^{*}$ : significativo estatisticamente para um $\mathrm{p} \leq 0,05$.

$$
\text { Fonte: Dados da pesquisa. }
$$

\section{Discussão}

No presente estudo não foram observadas diferenças de IM entre o sexo dos pacientes, diferindo de Passos et al (2012) e Bleich et al, (2009) que apontaram maior razão de chances para a ocorrência de potenciais IM em mulheres ${ }^{(14,15)}$. Já Cruciol-Souza et al (2006) identificaram que as IM prescritas em âmbito hospitalar acometem, na maioria, os homens $(61 \%)^{(16)}$. Na prevalência geral, observou-se em outro estudo que a maioria dos internados eram homens ${ }^{(17)}$, diferente do presente estudo em que a maioria eram mulheres.

Em relação à faixa etária, Passos et al (2012), verificaram maior número de IM em idosos, assim como verificado no presente estudo ${ }^{(14)}$. Este autor justificou pelo fato de que os idosos, em sua grande maioria, serem portadores de doenças crônicas, e consequentemente utilizarem um número superior de medicamentos, além de apresentarem, por vezes, redução das funções hepática e renal, o que compromete o metabolismo e eliminação dos fármacos, ainda aumenta as chances de $\mathrm{IM}^{(14)}$. Cruciol-Souza et al (2006) identificaram maior frequência de IM em adultos (média de idade 52,7 anos) $)^{(16)}$.

A média geral de idade do presente estudo foi semelhante à verificada por Gonçalves et al $(2016)^{(18)}$. Assim como a pesquisa de Melo (2010), 
desenvolvida em um hospital da Universidade de São Paulo, com média idade de 56,7 $\pm 19,8$ anos $^{(19)}$.

Quanto ao número de medicamentos prescritamos nesta pesquisa, foi observada média inferior aos demais estudos publicados referentes a este tema. Moreira et al, (2017), verificaram média de 9,3 $\pm 5,3$ fármacos/prescrição e Gonçalves et al (2016) média de 8,66 $\pm 4,37$ medicamentos/ prescrição (20,18). Em um estudo de Moura, Ribeiro e Magalhaes (2007) houve uma média de 6,4 medicamentos/ prescrição e o número de medicamentos variou de 2 a $14^{(21)}$, já, em outro estudo com medicamentos intravenosos, essa variação foi de 1 a 23 medicamentos ${ }^{(20)}$. O risco de IM aumenta com o número de medicamentos em uso, ocorrendo em $13 \%$ dos pacientes que tomam dois medicamentos e em $85 \%$ dos que utilizam mais de seis ${ }^{(14)}$.

O medicamento mais prescrito, verificado neste estudo, foi enalapril seguido de morfina e metoclopramida. Outro trabalho também verificou a metoclopramida entre os medicamentos mais prescritos, além de dipirona e heparina ${ }^{(21)}$. A metoclopramida também destacou-se no estudo de Silva et al $(2010)^{(1)}$.

Em relação à média de IM por pacientes, o resultado obtido neste estudo se aproxima do verificado por Passos et al (2012), que identificaram que a média de IM por paciente, variou de 2 a $11,6^{(14)}$. Os valores médios de IM também foram semelhantes nos estudos de Locatelli (2007) e Gonçalves et al (2016) entre pacientes hospitalizados ${ }^{(22,18)}$.

Quanto à classificação das IM, foi verificado nesse estudo maior frequência de IM graves, semelhante a outros estudos ${ }^{(23,1)}$. No estudo de Passos et al (2012) 33\% das IM foram classificadas como graves e $57 \%$ moderadas $^{(14)}$. No estudo de Junior et al (2009), as interações consideradas de gravidade moderada retrataram maioria dos casos $(55,5 \%)$, sendo representadas principalmente pelas interações captopril/dipirona sódica (17,5\%), furosemida/dipirona sódica $(8,2 \%)$ e captopril/furosemida $(5,9 \%)^{(24)}$. No estudo de Gonçalves (2016), a interação entre midazolan/ fentanil foi a mais frequente ${ }^{(18)}$. $\mathrm{O}$ alto índice de IM observadas indica a relevância desse tema em pacientes hospitalizados, a necessidade de avaliar e monitorar a terapêutica medicamentosa no sentido de prevenir e diminuir as consequências dos efeitos decorrentes de potenciais IM. Prescrições com alto risco de interações medicamentosas devem ser cuidadosamente monitoradas, visando à segurança do paciente (23). Em virtude da posição ocupada no sistema de utilização de medicamentos, o farmacêutico representa uma ligação crítica, logística e profissional entre o processo de distribuição de medicamentos e o de utilização. Além disso, tem importante participação na identificação e na correção de problemas potenciais e reais que envolvam medicamentos, trazendo benefícios para o paciente. A análise de prescrições de medicamentos realizada pelo farmacêutico, aliada à realização de intervenções acerca dos erros identificados, tem sido proposta como estratégia para aumentar a segurança no processo de uso de medicamentos ${ }^{(1)}$.

Quanto às classes de medicamentos mais suscetíveis às IM, no estudo de Passos et al (2012), as classes terapêuticas mais frequentes para IM foram: Anti-Hipertensivos, Anticoagulantes, Antibióticos e Analgésicos, sendo que os fármacos mais frequentes nas IM foram: Propranolol/Dipirona; Varfarina/Heparina, Propranolol/furosemida; Dipirona/Captopril ${ }^{(14)}$. No presente estudo a IM mais frequente foi entre os medicamentos morfina e tramadol. Em um estudo desenvolvido em uma Unidade de Terapia Intensiva, o medicamento tramadol apresentou potencial de interação com muitos medicamentos, como antibacterianos (por exemplo, o ciprofloxacino) e medicamentos que agem no Sistema Nervoso Central (por exemplo, a amitriptilina $)^{(1)}$. O tramadol e a metoclopramina também foram as mais frequentes em estudo realizado em uma UTI ${ }^{(17)}$.

Em um estudo realizado em ambulatório com idosos com câncer verificou-se associação de interações medicamentosas com idade maior que 70 anos, depressão, hipertensão arterial sistêmica, diabetes e artrite/artrose ${ }^{(25)}$. No presente trabalho observou-se associação entre interações medicamentosas e hipertensão, porém diagnóstico de diabetes não foi associado à presença de interações medicamentosas, assim como o estudo de Santos $(2017)^{(7)}$. Em estudo em um hospital universitário, foi observado que tanto hipertensão arterial sistêmica quanto diabetes mellitus foram 
fatores de risco para ocorrência de $\mathrm{IM}^{(19)}$.

No presente estudo foi observado associação entre o uso de anti-hipertertensivos, das classes beta bloqueadores e do sistema renina angiotensina, e apresentar interações medicamentosas, porém não foram encontrados trabalhos que avaliassem essa associação. Os anti-hipertensivos foram a classe mais citada no estudo de interações medicamentosas de Passos et al (2012) ${ }^{(14)}$, já citado, além disso, esses medicamentos têm uso elevado, considerando a grande prevalência de hipertensos, o que justifica a maior porcentagem de potenciais interações.

Verificou-se que o sistema mais acometido por IM foi o Sistema Nervoso Central, assim como outros trabalhos ${ }^{(7,18)}$. Observou-se também que o uso de psicolépticos e psicoanalépticos estavam associados à presença de interações medicamentosas, tais dados também foram observados por Santos $(2017)^{(7)}$. O uso de corticoesteróides sistêmicos também mostrou associação com IM, mas também não foram encontrados outros estudos que fizessem tal comparação. Entretanto, foi identificado em estudo de incompatibilidades medicamentosas de um hospital universitário, em que a hidrocortisona, um medicamento da classe dos corticoides, foi o segundo mais envolvido nas incompatibilidades medicamentosas ${ }^{(26)}$.

É crescente a preocupação com fármacos que possuem a propriedade de prolongar o intervalo QT, devido ao risco de cardiotoxicidade com torsade de points e parada cardíaca. Esses eventos adversos podem ser determinados por potenciais interações farmacocinéticas que inibem o metabolismo de fármacos com essa propriedade ou por sinergismo farmacodinâmico ${ }^{(27)}$. Embora no presente estudo o sistema mais acometido por IM tenha sido o Sistema Nervoso Central, o Sistema Cardiovascular também se destacou e esse dado é relevante para ações que possam mitigar a ocorrência de IM.

Deve-se ressaltar que as interações medicamentosas avaliadas no presente estudo foram em potencial. Embora nem todas as interações medicamentosas possam ser prevenidas e efetivamente ocorram com o paciente, faz-se necessária propagação do conhecimento entre os profissionais de saúde, por meio do profissional farmacêutico ${ }^{(28)}$. Uma das atividades desenvolvidas por esse profissional na atuação clínica é a avaliação das prescrições médicas, a posologia, a interação do medicamento com outros fármacos, com alimento ou com alguma patologia, a via de administração, a indicação terapêutica e os efeitos adversos no intuito de prevenir e resolver os problemas relacionados a medicamentos ${ }^{(29)}$.

Nesse interim, uma IM pode comprometer a eficácia ou a segurança do paciente, prolongar o período de internação, aumentar os custos hospitalares e principalmente comprometer a qualidade de vida do paciente. $\mathrm{O}$ acompanhamento do farmacêutico se faz imprescindível na prevenção e no monitoramento da terapêutica ${ }^{(22)}$.

\section{Considerações finais}

No presente estudo, destacou-se a necessidade da avaliação quanto ao uso racional de medicamentos e à presença de IM nas prescrições hospitalares. A IM mais frequente foi entre a Morfina e o Tramadol (7,7\%). A média de

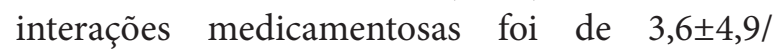
paciente, e a média de medicamentos prescritos foi $3,1 \pm 1,8 /$ paciente. Observou-se que os pacientes hipertensos estavam mais suscetíveis a IM. O conhecimento dos profissionais de saúde, principalmente prescritores, sobre IM pode evitar que sejam prescritos medicamentos que interajam entre si, ou minimizar o impacto negativo por meio da monitorização adequada, quando a combinação for inevitável.

A atuação do farmacêutico pode constituir um dos principais instrumentos de prevenção das IM, contribuindo para uma assistência de qualidade e livre de danos para o paciente, e impactando de forma positiva na melhoria da qualidade de vida no que se refere à otimização da farmacoterapia e o uso racional de medicamentos.

\section{Referências}

1. SILVA, N.M.O. et al. Avaliação de potenciais interações medicamentosas em prescrições de pacientes internadas, em hospital público universitário especializado em saúde da mulher, em Campinas-SP. Revista de Ciências Farmacêuticas Básica e Aplicada. 2010;31(2):171-176. 
2. PEREIRA, G.J.S. et al. Estudo de utilização de medicamentos na clínica médica. Rev. Bras. Farm. 2008;3(89):267-71.

3. PINTO, I.V.L.; CASTRO, M.S.; REIS, A.M.M. Descrição da atuação do farmacêutico em equipe multiprofissional com ênfase no cuidado ao idoso hospitalizado. Rev. Bras. Geriatr. Gerontol., Rio de Janeiro. 2013;4(16):747-758.

4. DANTAS, M.; SANTOS, V. Implicações da polifarmácia em idosos e a contribuição da assistência farmacêutica. Leituras: Educação Física e Esportes [Internet]. 2018. 23 (240):87-9.

5. FERREIRA, S.F. et al. Avaliação de interações medicamentosas em prescrições de pacientes hospitalizados. Revista Racine.2006;16(94):67-70.

6. CEDRAZ, K.N.; SANTOS, J.M.C. Identificação e caracterização de interações medicamentosas em prescrições médicas da unidade de terapia intensiva de um hospital público da cidade de Feira de Santana, BA Rev Soc Bras Clin Med. 2014;12(2):124-30.

7. SANTOS, M.H.B.A. Análise de interações medicamentosas potenciais e de eventos adversos a medicamentos em uma unidade de terapia intensiva. Tese (Mestrado em Epidemiologia em Saúde Pública) - Escola Nacional de Saúde Pública Sergio Arouca, na Fundação Oswaldo Cruz, Rio de Janeiro, p. 60, 2017.

8. SANTOS, M.T. Variáveis associadas a risco de incompatibilidades medicamentosas em prescrições de crianças e adolescentes portadores de neoplasia. Tese (Mestrado em Saúde da Criança e do Adolescente) - Universidade Federal do Rio Grande do Sul, Porto Alegre, p. 5, 2017.

9. CORRER, C.J. et al. Riscos de problemas relacionados com medicamentos em pacientes de uma instituição geriátrica. Rev Bras Ciênc Farm. 2007;1(43):55-62.

10. BITTENCOURT, V.L.L. et al. Factors associated with the risk of falls in hospitalized adult patients. Rev Esc Enferm USP. 2017;51:e03237. DOI: http://dx.doi.org/10.1590/ S1980-220X2016037403237

11. WHO Collaborating Centre for Drug Statistics Methodology, ATC/DDD Index. [Última atualização 2009 dez 14; acesso em 2018 set 10]. Disponível em: https://www.whocc.no/atc_ddd_index/

12. Micromedex (r) Healthcare Series [Internet]. Greenwood Vilage: Thomson Healthcare; 2016 [acesso em 2018 out 22]. Disponível em: www.micromedex.com

13. PORTO C.C. et al. Interação medicamentosa. Rio de Janeiro: Guanabara Koogan, 2010.

14. PASSOS M.M.B. et al. Interações medicamentosas em pacientes internados na clínica médica de um hospital de ensino e fatores associados. Rev. Bras. Farm. 2012; 93(4):450-456.
15. BLEICH G.W. et al. Frequency of potential interactions between drugs in medical prescriptions in a city in southern Brazil. São Paulo Medical Journal. 2009;127(4);206-210.

16. CRUCIOL-SOUZA J.M.; THOMSON J.C. Prevalence of potential drug-drug interactions and its associated factors in a Brazilian teaching hospital. J Pharm Pharm Sci. 2006; 9(3):427-433.

17. SILVA U.D.A. et al. Interações medicamentosas e consequentes intervenções farmacêuticas na Unidade de Terapia Intensiva de um hospital privado em Macapá, Amapá. Vigil. sanit. Debate. 2018;6(2):29-37.

18. GONÇALVES S.S. et al. Ocorrência clínica de interações medicamentosas em prescrições de pacientes com suspeita de reação adversa internados em um hospital no interior da Bahia. Revista de Atenção à Saúde. 2016;14(48), 32-39.

19. Melo DO. Avaliação das interações potenciais para pacientes internados na clínica médica do hospital universitário da USP visando à elaboração de instrumento para identificação de eventos adversos a medicamentos evitáveis. Tese (Doutorado em Fármaco e Medicamentos) - Faculdade de Ciências Farmacêuticas, Universidade de São Paulo, p. 49, 2010.

20. MOREIRA M.B. et al. Potential intravenous drug interactions in intensive care. Rev Esc Enferm USP 2017;51:e03233. DOI: http://dx.doi.org/10.1590/S1980220X2016034803233

21. MOURA C.S.; RIBEIRO A.Q.; MAGALHÃES S.S. Avaliação de interações medicamentosas potenciais em prescrições médicas do Hospital das Clínicas da Universidade Federal de Minas Gerais (Brasil). Latin american journal of pharmacy. 2007; 26(4):596-601.

22. LOCATELLI J. Interações medicamentosas em idosos hospitalizados. Rev. Einstein On Line, 2007; 5(4):343346.

23. VONBACH P. et al. Prevalence of drug-drug interactions at hospital entry and during hospital stay of patients in internal medicine. Eur J Intern Med. 2008; 19(6):413-420.

24. PIVATTO F.P. et al. Potenciais interações medicamentosas em prescrições de um hospital-escola de Porto Alegre. Rev Assoc Med Rio Grande Do Sul. 2009; 53(3): 251-6.

25. Faria C.D.O. et al. Interações Medicamentosas na Farmacoterapia de Idosos com Câncer atendidos em um Ambulatório de Onco-Hematologia. Rev. bras. Cancerol. 2018;64(1):61-68.

26. MARSILIO N.R.; SILVA D.S.; BUENO D.B. Incompatibilidades medicamentosas em centro de tratamento intensivo adulto de um hospital universitário. Revista brasileira de terapia intensiva. 2016;28(2):147153. 
27. CARVALHO R.E.F.L. et al. Prevalência de interações medicamentosas em unidades de terapia intensiva no Brasil. Acta Paul Enferm. 2013;2(26):150-157.

28. NEVES, C; COLET, C. Perfil de uso de antimicrobianos e suas interações medicamentosas em uma UTI adulto do Rio Grande do Sul. Revista de Epidemiologia e Controle de Infecção. 2015;5(2):65-71.

29. MIRANDA T.M.M. et al. (2012). Intervenções realizadas pelo farmacêutico clínico na unidade de primeiro atendimento. Einstein (São Paulo). 2012;10(1):74-78.

\section{Como citar este artigo:}

Petri AA, Schneider A, Kleibert KRU, Bittencourt VLL, Winkelmann ER, Colet CF. Interações medicamentosas potenciais em pacientes hospitalizados. Rev. Aten. Saúde. 2020; 18(63): 31-42. 